\title{
Effects of tin phosphate nanosheet addition on proton-conducting properties of sulfonated poly(ether sulfone) membranes
}

\author{
Shoichi Sugata a,*, Shinya Suzuki ${ }^{a}$, Masaru Miyayama a ${ }^{a}$, Enrico Traversa b,c,**, Silvia Licoccia ${ }^{b}$ \\ a Research Center for Advanced Science and Technology, The University of Tokyo, 4-6-1 Komaba, Meguro-ku, Tokyo 153-8904, Japan \\ ${ }^{\mathrm{b}}$ Department of Chemical Science and Technology \& NAST Center, University of Rome Tor Vergata, Via della Ricerca Scientifica, Rome 00133, Italy \\ c International Center for Materials Nanoarchitectonics (MANA), National Institute for Materials Science (NIMS), 1-1 Namiki, Tsukuba, Ibaraki 305-0044, Japan
}

\section{A R T I C L E I N F O}

\section{Article history:}

Received 4 April 2012

Received in revised form 27 August 2012

Accepted 30 August 2012

Available online 30 September 2012

\section{Keywords:}

Organic/inorganic nanocomposite

Tin phosphate nanosheet

Proton conductivity

Fuel cell

\begin{abstract}
A B S T R A C T
Organic/inorganic composite membranes were prepared by dispersing nanosheets of layered tin phosphate hydrate $\left[\mathrm{Sn}\left(\mathrm{HPO}_{4}\right)_{2} \cdot n \mathrm{H}_{2} \mathrm{O}(\mathrm{SnP})\right]$ in sulfonated poly(ether sulfone) (SPES) at SnP contents of 0-40 vol.\%. The stabilities and proton conductivities of SPES/SnP nanosheet (SnP-NS) composite membranes were investigated and compared with those of SPES/SnP particle (SnP-P) composite membranes. The chemical stabilities as evaluated by thermogravimetry, differential thermal analysis, and diffuse reflectance Fourier-transform infrared spectroscopy were improved in both composite membranes. The improvement in the structural stability of SPES/SnP-NS composite membranes was more evident than that in SPES/SnP-P. The results suggest that exfoliation of SnP increases the area of the SPES-SnP interface and extends the connectivity of the network of hydrogen bonds. A composite membrane containing 10 vol.\% SnP-NS (SPES/SnP-NS10vol.\%) showed a high conductivity of $5.9 \times 10^{-2} \mathrm{~S} \mathrm{~cm}^{-1}$ at $150{ }^{\circ} \mathrm{C}$ under saturated water vapor pressure. Although less water was present in SPES/SnP-NS10vol.\% than in SPES/SnP-P10vol.\% or pure SPES, the conductivity of SnP-NS10vol.\% was the highest among these samples at $130{ }^{\circ} \mathrm{C}$ under a high relative humidity $(\mathrm{RH})$. However at a low $\mathrm{RH}$, the proton-conducting property was not improved by changing the composition of the SnP-NS. These results suggest that the hydrogen-bond network operates effectively for proton conduction at a high $\mathrm{RH}$, but at a low $\mathrm{RH}$, the network fails to conduct as a result of a decrease in water content accompanied by structural stabilization.
\end{abstract}

(c) 2012 Elsevier B.V. All rights reserved.

\section{Introduction}

The proton-exchange membrane fuel cell (PEMFC) shows promise for high-efficiency energy conversion as a clean source of alternative energy for transportation, stationary power devices, or portable electrical devices. However, the PEMFC has several problems, such as poisoning of the platinum-based catalyst by carbon monoxide present in the reformed fuel gas, a decrease in the effective area of the electrodes by liquid water produced in the working cell, and heat-exchange requirements [1]. Most of these problems can be solved by operating the PEMFC at intermediate temperatures above $100{ }^{\circ} \mathrm{C}$. Commonly used perfluorosulfonic acid polymers such as Nafion ${ }^{\circledR}$ show outstanding electrochemical and physicochemical properties, but the thermal resistance of these polymer membranes is inadequate for long-term use at such intermediate temperatures.

\footnotetext{
* Correspondence to: Enrico Traversa, Research Center for Advanced Science and Technology, The University of Tokyo, 4-6-1 Komaba, Meguro-ku, Tokyo 153-8904, Japan.

** Corresponding author. Tel.: +8135452 6555; fax: +81354525083.

E-mail address: sugata@crm.rcast.u-tokyo.ac.jp (S. Sugata).
}

Many researchers are examining alternative nonperfluorinated polymers for fuel-cell applications, such as sulfonated poly(ether ether ketone)s (SPEEKs), sulfonated poly(ether sulfone)s (SPESs), sulfonated polybenzimidazoles, and sulfonated polyimides [2-6]. Such polymers can be rendered proton conducting by functionalization with sulfonic acid $\left(\mathrm{SO}_{3} \mathrm{H}\right)$ groups. It is known that sulfonic acid $\left(\mathrm{SO}_{3} \mathrm{H}\right)$ groups in SPES have a high acidity because of the stronger electron-withdrawing nature by the sulfonate group in comparison with those in other sulfonated hydrocarbon polymers, which leads to a high proton conductivity $[7,8]$. However, high levels of sulfonation are accompanied by swelling of the polymer or water solubility; furthermore, the proton conductivity decreases markedly in conditions of low relative humidity (RH) as a result of dehydration [2,3]. It is therefore necessary to develop membranes that have a high chemical stability and a high proton conductivity at intermediate temperatures under conditions of low RH.

One possible approach to solving these problems is to add an inorganic compound to the organic matrix [9-14]. It has been reported that the chemical, thermal, and mechanical stabilities of membranes can be improved by dispersing an inorganic filler in the organic matrix. It has also been reported that rapid ionic transfer through the interface between the two different materials occurs in some proton-conducting organic/inorganic composite materials [12,15]. Higher stabilities, greater proton conductivity, or better fuel-cell performance have been reported 
for some composite systems such as Nafion $/ \mathrm{TiO}_{2}, \mathrm{SPEEK} / \mathrm{WO}_{3}$, SPES/ heteropoly acid $\left(\mathrm{H}_{3} \mathrm{PW}_{12} \mathrm{O}_{40}\right)$, SPES/boron phosphate $\left(\mathrm{BPO}_{4}\right)$, or SPEEK/ organically treated montmorillonite (OMMT) [12-14,16-19].

Layered metal phosphate hydrates $\mathrm{M}\left(\mathrm{HPO}_{4}\right)_{2} \cdot n \mathrm{H}_{2} \mathrm{O}(\mathrm{M}=\mathrm{Sn}, \mathrm{Zr}, \mathrm{Ti})$ (MP) are layered compounds with water molecules in the interlayer that show high proton conductivities $[20,21]$. Among these hydrates, $\mathrm{Sn}\left(\mathrm{HPO}_{4}\right)_{2} \cdot n \mathrm{H}_{2} \mathrm{O}(\mathrm{SnP})$ shows a high and stable proton conductivity above $100{ }^{\circ} \mathrm{C}$ [20]. The composite membranes SPEEK/SnP and SPES/ SnP have been reported to show thermal stabilities and proton conductivities that are superior to those of the corresponding pure polymers at temperatures up to $150{ }^{\circ} \mathrm{C}[22,23]$. It has been confirmed that the presence of hydrogen bonds at the organic-inorganic interface in these composites improves their thermal stabilities, and that connectivity between the hydrogen-bond networks improves their proton conductivity. Moreover, an SPES/SnP membrane showed a high conductivity of $2.6 \times 10^{-3} \mathrm{~S} \mathrm{~cm}^{-1}$ at $130{ }^{\circ} \mathrm{C}$ under $40 \% \mathrm{RH}$ [22].

Several layered compounds can be exfoliated to form nanosheets by several methods; these nanosheets are two-dimensional crystals with a lateral size of $100 \mathrm{~nm}$ to $10 \mu \mathrm{m}$ and a thickness of 1-10 nm [24-26]. The organic-inorganic interfacial area between polymers and layered inorganic materials can therefore be markedly increased by exfoliation of the inorganic material. Nafion/ZrP-nanosheet (ZrP-NS) membranes have been reported to show enhanced chemical stabilities without any diminution of their proton conductivities [27]. However, their proton-conducting properties at intermediate temperatures have not yet been reported. It has been reported that SPEEK/titania nanosheet (TNS) composites with a very low TNS contents show higher proton conductivities than does pure SPEEK, because of the surface properties of the inorganic filler [28]. It has also been reported that the large area of the SPEEK/ZrP-NS interfaces results in an improvement in the proton conductivity of the composites at intermediate temperatures and in their dry resistance [29]. We therefore focused our attention on SnP nanosheet (SnP-NS) as an inorganic filler material and SPES as an organic matrix.

We investigated the thermal and chemical stabilization of SPES/ SnP-NS membranes through formation of hydrogen-bonded networks with SnP and we examined the proton-conducting properties of SPES/ SnP-NS composite membranes under various conditions of $\mathrm{RH}$. On the basis of our results, we discuss the proton-conduction mechanism and the possibility of using SPES/SnP-NS membranes as electrolytes for PEMFCs operating at intermediate temperatures.

\section{Experimental}

\subsection{Fabrication of membranes}

SPES was synthesized by direct, aromatic nucleophilic substitution, stepwise polymerization of bisphenol A (4,4'-propane-2,2-diyldiphenol; Tokyo Chemical Industry Co. Ltd., Tokyo), bis(4-chlorophenyl sulfone) (DCDPS, Tokyo Chemical Industry), and 3,3'-disulfonated-4,4'dichlorodiphenyl sulfone (SDCDPS). SDCDPS was synthesized from DCDPS by sulfonation with fuming sulfuric acid $\left(30 \% \mathrm{SO}_{3}\right.$, Wako Pure Chemical Industries Ltd., Osaka). The detailed procedure for polymerization has been described in earlier reports $[7,8]$. The average number of $-\mathrm{SO}_{3} \mathrm{H}$ groups per repeat unit of the polymer [the degree of sulfonation (DS)] was determined by titration to be 1.0 .

SnP particles (SnP-P) were synthesized by a hydrothermal reaction as described elsewhere [20]. SnP-P was exfoliated into nanosheets by using an aqueous solution of tetrabutylammonium hydroxide (TBAOH, Tokyo Chemical Industry) [29]. A $0.3 \mathrm{M}$ solution of TBAOH was prepared by titration with $85 \%$ phosphoric acid (Wako) to a constant $\mathrm{pH}$ of 7. Colloidal suspensions of SnP-NS were prepared by stirring SnP-P $(6.0 \mathrm{mmol})$ in the TBAOH solution $(200 \mathrm{~mL})$ at room temperature for 1 week. The resulting SnP-NS were isolated by centrifugation and washed repeatedly with deionized water to remove residual
TBAOH and $\mathrm{H}_{3} \mathrm{PO}_{4}$, then dispersed in deionized water by agitation. The concentration of $\mathrm{Sn}$ in the dispersion was measured by inductively coupled plasma atomic emission spectroscopy (SPS3100; SII NanoTechnology Inc., Chiba).

The proper amount of $\mathrm{SnP}$ was dispersed in $\mathrm{N}, \mathrm{N}$-dimethylacetamide (DMA; Wako) and irradiated with ultrasound for $24 \mathrm{~h}$. SPES was mixed and dissolved in the DMA suspension of SnP. After mixing and defoaming in a conditioning mixer (ARE250; Thinky, Tokyo), the mixture was cast onto a Teflon plate and kept at $80{ }^{\circ} \mathrm{C}$ overnight. The resulting membranes were immersed in $1 \mathrm{M}$ aqueous $\mathrm{H}_{2} \mathrm{SO}_{4}$ for $24 \mathrm{~h}$, washed with deionized water, and dried at $80{ }^{\circ} \mathrm{C}$ for $48 \mathrm{~h}$. The SnP contents of the resulting membranes were $0-50$ vol.\% and their thicknesses were 150-300 $\mu \mathrm{m}$. The crystal structures of SPES/SnP-P and SPES/SnP-NS were evaluated by X-ray diffraction (XRD) using $\mathrm{Cu}$ $K \alpha$ radiation (Bruker $\mathrm{AXS}$ ) at room temperature.

\subsection{Characterization of membranes}

Thermogravimetric analysis (TGA) and differential thermal analysis (DTA) were performed with a Thermoplus TG8120 (Rigaku, Tokyo) at a heating rate of $10{ }^{\circ} \mathrm{C} / \mathrm{min}$ under dry air. Fourier-transform infrared (FT-IR) spectra of the prepared membranes were recorded at room temperature on an IR Prestage-21 (Shimadzu, Kyoto). The spectra were measured as the average of 100 scans with a resolution of $2 \mathrm{~cm}^{-1}$. The microstructure of the prepared membranes was observed by scanning electron microscopy (SEM) with a Hitachi S-4500 instrument (Hitachi Ltd., Tokyo). The membranes were fractured by brief immersion in liquid nitrogen and the resulting samples were vacuumsputtered with 20-nm layer of Au for analysis. The water uptake (W.U.) of the prepared membranes as a mass percentage was estimated by means of the following equation:

$W . U .(\mathbf{w t} \%)=\left[\left(W_{\text {wet }} / W_{\text {dry }}\right)-1\right] \times 100$

where $W_{\text {wet }}$ is the weight of the wet membrane and $W_{\text {dry }}$ is the weight of the dry membrane. The wet membranes were prepared by immersing the dry membranes in water at $30{ }^{\circ} \mathrm{C}$ for $48 \mathrm{~h}$. Surface water was then wiped off the wet membranes with absorbent paper, and the wet membranes were weighted.

The conductivity was measured by the impedance method over a frequency range of $5 \mathrm{~Hz}$ to $13 \mathrm{MHz}$ at an applied voltage of $0.1 \mathrm{~V}$ by using a 4192A impedance analyzer (Hewlett Packard, Palo Alto, CA). Silver electrodes were applied to the surface of the membranes by painting on as a paste and drying at $60{ }^{\circ} \mathrm{C}$. The samples were placed in a stainless vessel at $40-150{ }^{\circ} \mathrm{C}$, and the water vapor pressure around the samples was controlled at 0-0.5 MPa by regulating a leak valve or by introducing water vapor from a separate vessel. The temperature of the sample was monitored by using a thermocouple placed close to the sample.

Isothermal desorption of water was measured at various external water-vapor pressures from $95 \%$ relative humidity $(\mathrm{RH})$ to dry conditions $(<2 \% \mathrm{RH})$ at $130{ }^{\circ} \mathrm{C}$ by using a pressure-resist thermogravimetric measurement system (Nihon Bell Co., Ltd.) in which the vapor pressure of water is controlled as previously described [30]. The RH was estimated by using the expression $P_{\mathrm{H} 2 \mathrm{O}} / P_{\mathrm{S}}$ where $P_{\mathrm{H} 2 \mathrm{O}}$ is the vapor pressure of water in the vessel and $P_{\mathrm{s}}$ is the saturated water vapor pressure at $130{ }^{\circ} \mathrm{C}$.

\section{Results and discussion}

In our previous report on SPES/SnP-P composite membranes [22], the DS of the prepared polymer was 1.2. However, because of the aggregation of SnP-NS caused by the high acidity of SPES (DS $=1.2$ ), it was difficult to prepare homogeneous composite membranes based on SPES ( $D S=1.2$ ) and SnP-NS. We therefore prepared composite 
membranes of SPES (DS $=1.0$ ) and SnP, and we examined their properties.

The XRD patterns of the composite membranes containing 10 vol.\% SnP-P and SnP-NS are shown in Fig. 1. The diffraction peaks can be indexed to $\mathrm{Sn}\left(\mathrm{HPO}_{4}\right)_{2} \cdot \mathrm{H}_{2} \mathrm{O}$ (JCPDS 31-1397). Small amounts of an adhesive material were detected as an impurity around $2 \theta=26$ and $29^{\circ}$. The structure of SnP-NS was not altered during the exfoliation reaction of SnP.

The SnP particles had a plate-like shape with a size of 150-200 nm, a thickness of 25-30 nm, and a specific surface area of $30-35 \mathrm{~m}^{2} \mathrm{~g}^{-1}$, as previously reported [22]. Fig. 2 shows an atomic force microscopy (AFM) image of exfoliated SnP-NS deposited on a mica substrate. The thickness and lateral size of the exfoliated nanosheets were $1-5 \mathrm{~nm}$ and $50-100 \mathrm{~nm}$, respectively. The specific surface area, roughly estimated from the AFM image, was $200-300 \mathrm{~m}^{2} \mathrm{~g}^{-1}$.

SEM images of the composite membranes containing 10 vol.\% SnP-P and SnP-NS are shown in Fig. 3. The SEM image (a) shows that a relatively homogeneous dispersion of $\mathrm{SnP}$ particles was achieved throughout the SPES matrix. The SEM image (b) shows that some SnP nanosheets aggregated in SPES/SnP-NS. It is probable that SnP nanosheets restacked in the DMA suspension of SPES/SnP-NS because of the acidity of SPES. However the aggregated nanosheets did not sink into the bottom of the composite membrane and they were dispersed in a similar level to the SPES/SnP-P composite membrane.

Fig. 4 shows the water uptake of the prepared membranes. The water uptake decreased with increasing SnP content in both the SPES/SnP-P and SPES/SnP-NS composites. These decreases were much larger than those estimated from the volume decrease of SPES. The degree of decrease in SPES/SnP-NS membranes was greater than that in SPES/SnP-P membranes.

It has been reported that proton conductivity increases or decreases depending on the content of SnP in SPEEK/SnP composite membrane [23]. It is assumed that a SPEEK-SnP interfacial layer and a SPEEK-SnP interfacial region outside the SPEEK-SnP interfacial layer are formed around each particles of SnP. Because SPEEK-SnP interfacial layers contain high concentrations of hydrogen bonds, their chemical stability is improved and their proton conductivity is high as a result of the presence of networks of hydrogen bonds. On the other hand, the SPEEK-SnP interfacial region has an improved chemical stability as a result of interfacial effects, but its proton conductivity is low as a result of its low water content. In the case of lower contents of SnP (up to 25 vol.\%),

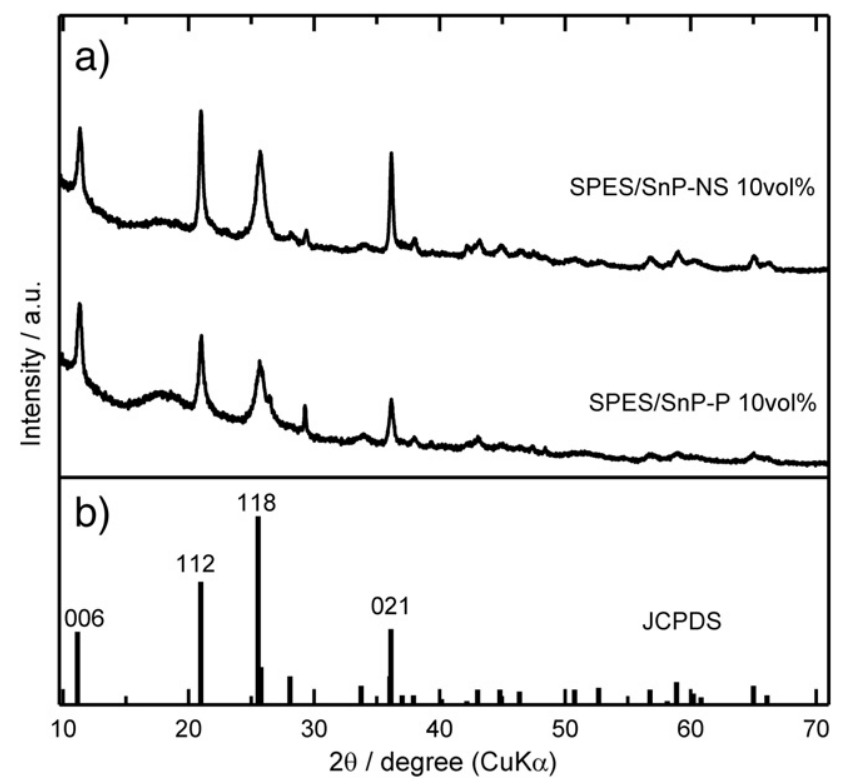

Fig. 1. (a) XRD patterns of the composite membranes containing 10 vol.\% SnP-P and SnP-NS, and (b) the peak assignment in JCPDS 31-1397 for $\mathrm{Sn}\left(\mathrm{HPO}_{4}\right)_{2} \cdot \mathrm{H}_{2} \mathrm{O}$.
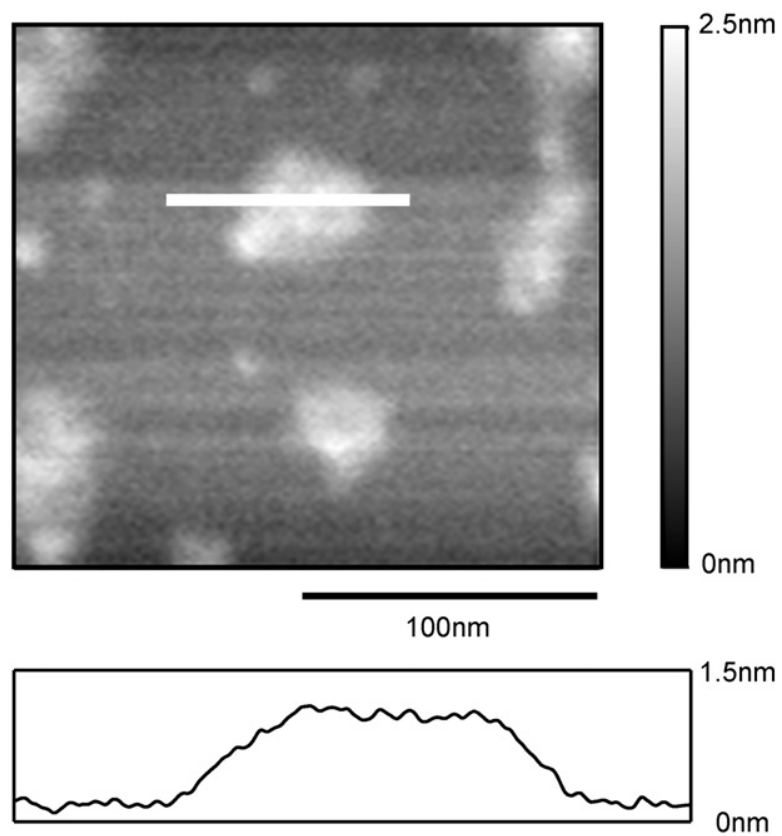

Fig. 2. Atomic-force microscopy images of SnP-NS. The upper image shows the two-dimensional diagram, and the lower image shows a cross-sectional diagram at the line depicted in the upper image.

the area of the SPEEK-SnP interfacial region increases with increasing SnP content, but the interfacial layers are not connected. As a result, the proton conductivity decreases as a result of discontinuities in the proton-conducting pathway. When large amounts of SnP (above 25 vol.\%) are added to the SPES matrix, the proton conductivity
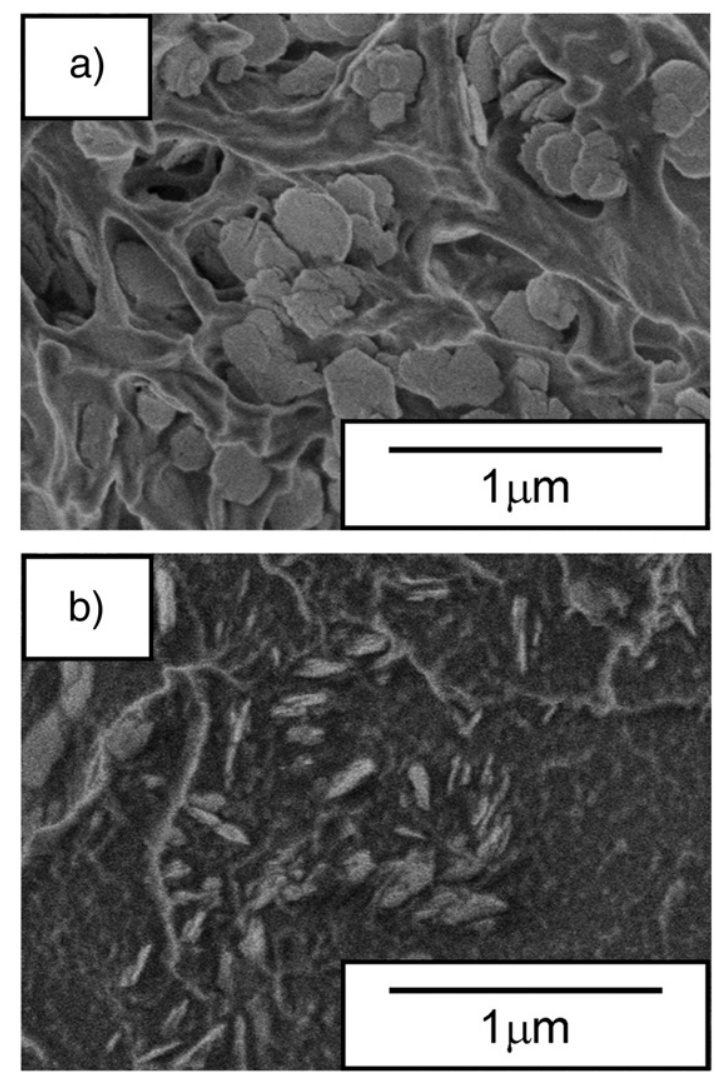

Fig. 3. SEM images of the composite membranes containing 10 vol.\% SnP-P (a) and SnP-NS (b). 


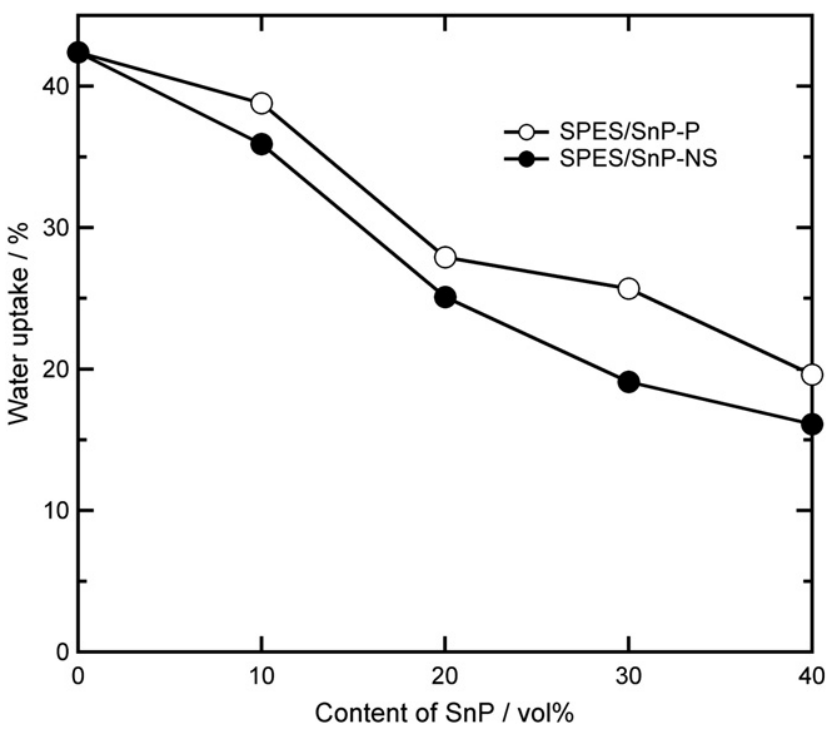

Fig. 4. Water uptakes of SPES/SnP membranes with various SnP contents.

improves as a result of the connectivity of the interfacial layer, which operates as a proton-conducting path.

We assumed that the decrease in water uptake of the composite membranes shown in Fig. 4 is the result of stabilization of the microstructure at the SPES-SnP heterointerface by the hydrogen-bond network, as reported for SPEEK/SPES composite membranes [23]. The effect of suppression of swelling produced by the addition of SnP-NS was more evident than that produced by the addition of SnP-P. We suggest that the effect in improving the structural stability is increased as a result of an increase in the SPES-SnP interfacial area.

Fig. 5 shows the FT-IR spectra of the pure SPES and the composite membranes containing 10-40 vol.\% SnP-NS. In the FT-IR spectra, peaks assigned to symmetric $\mathrm{SO}_{3}^{-}$stretching vibrations appeared at $1022 \mathrm{~cm}^{-1}$ for the pure SPES membrane and the peak shifted to higher wavenumbers by the addition of SnP to the SPES matrix [31,32]. Fig. 6 shows the presence of peaks with wavenumbers of around $1030 \mathrm{~cm}^{-1}$ for the SPES/SnP-P and SPES/SnP-NS composite membranes. The wavenumbers of these peaks for both membranes increased with increasing SnP content. It has been reported that the

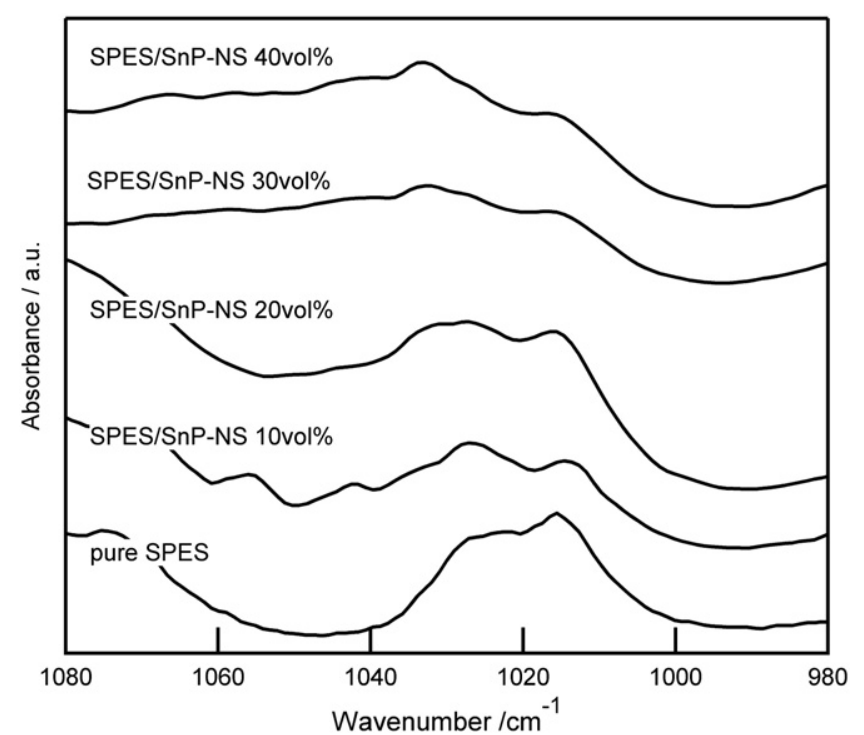

Fig. 5. FTIR spectra of pure SPES membrane and the composite membranes containing 10-40 vol.\% SnP-NS. microstructure of the organic-inorganic membranes is stabilized by the formation of hydrogen bonds between $-\mathrm{SO}_{3} \mathrm{H}$ groups in sulfonated polymers and interlayer water of layered inorganic fillers [12,33,34]. Fig. 6 suggests that SPES/SnP membranes contain stronger hydrogen bonds than do membranes of pure SPES. The peak shift for SPES/ SnP-NS membranes was larger than that for SPES/SnP-P membranes, indicating that the effect of stabilization was increased by the enlargement of the SPES-SnP interfacial area.

Several weight-loss steps were observed from TG curves (not shown here). On the basis of previous studies on SPEEK-based membranes $[6,12]$, we attributed the weight losses at $290-310{ }^{\circ} \mathrm{C}$ to the elimination of the $\mathrm{SO}_{3} \mathrm{H}$ groups from SPES chain, and we defined the temperature as the splitting-off temperature $\left(T_{s}\right)$ hereafter. Fig. 7 shows the dependence of the glass-transition temperature $\left(T_{g}\right)$ and $T_{s}$ on the SnP content for SPES/SnP membranes as measured by TGA. The $T_{g}$ and the $T_{s}$ of both membranes increased with increasing SnP content, and the degree of increase was greater for SPES/SnP-NS than for SPES/SnP-P. It has been reported that organic/inorganic composite structures are stabilized by hydrogen bonds formed between $\mathrm{SO}_{3} \mathrm{H}$ groups in a sulfonated polymer and interlayer water of a layered inorganic compound [12,34]. From the results of water uptake, FT-IR spectra $\mathrm{T}_{\mathrm{g}}$ and $\mathrm{T}_{\mathrm{s}}$, it can be concluded that the presence of a network of hydrogen bonds at the SPES-SnP interface improves the thermal stability and that enlargement of the interfacial area leads to further enhancement of the thermal stability.

Fig. 8 shows the temperature dependence of the conductivity of SPES/SnP-NS membranes with various SnP contents measured under saturated water vapor pressure. The composite membrane containing 10 vol.\% SnP-NS (SPES/SnP-NS10vol.\%) showed higher conductivities than pure SPES or SPES/SnP-P10vol.\% and the conductivity of SPES/ SnP-NS10vol.\% at $150{ }^{\circ} \mathrm{C}$ was $5.9 \times 10^{-2} \mathrm{~S} \mathrm{~cm}^{-1}$. SPES/SnP-NS20vol.\% showed a higher conductivity than pure SPES at above $110{ }^{\circ} \mathrm{C}$, but showed a lower conductivity at $100{ }^{\circ} \mathrm{C}$ or below. The slopes of conductivity plots for all samples became steeper above $100{ }^{\circ} \mathrm{C}$. We consider that the temperature dependence of apparent conductivity in the composite membranes include contributions of usual conductivity (product of concentration and mobility of protons from the sulfonate group) and the change of water content. Usually, the activation energy depends on the water content in the electrolyte [35]. Their change of slope is assumed to be due to the decrease in water content with increasing temperature below $100{ }^{\circ} \mathrm{C}$.

The dependence of the conductivity on the SnP content measured at $130{ }^{\circ} \mathrm{C}$ under $90 \%$ or $30 \% \mathrm{RH}$ for SPES/SnP membranes is shown in Fig. 9. Under $90 \% \mathrm{RH}$, the conductivities for SPES/SnP-NS membranes were higher than those for SPES/SnP-P membranes at the same SnP content. Under 90\% RH, SPES/SnP-NS10vol.\% showed the highest

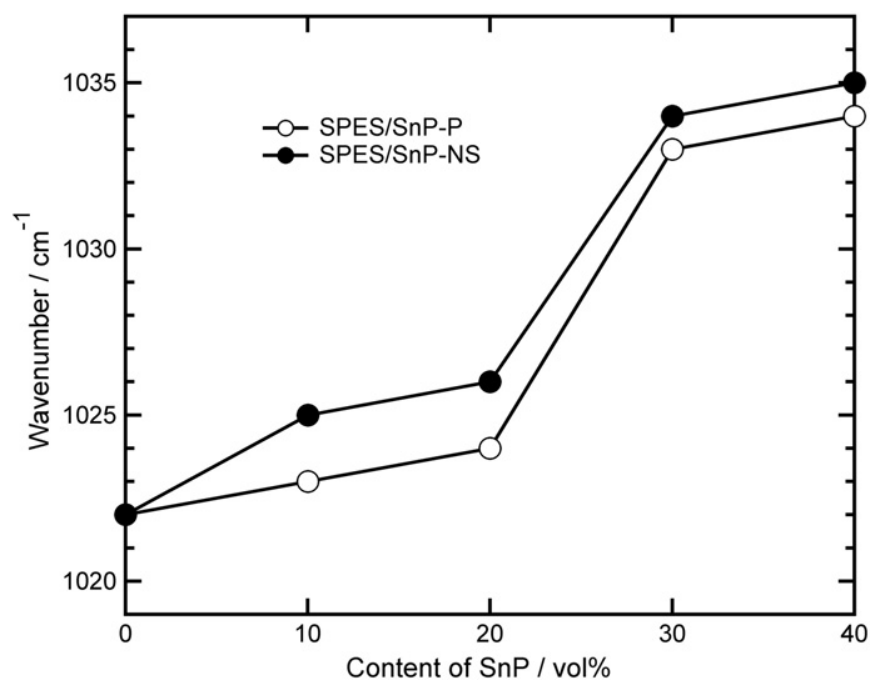

Fig. 6. Wavenumbers of the $\mathrm{SO}^{3-}$ bands of SPES/SnP membranes observed in FT-IR spectra. 


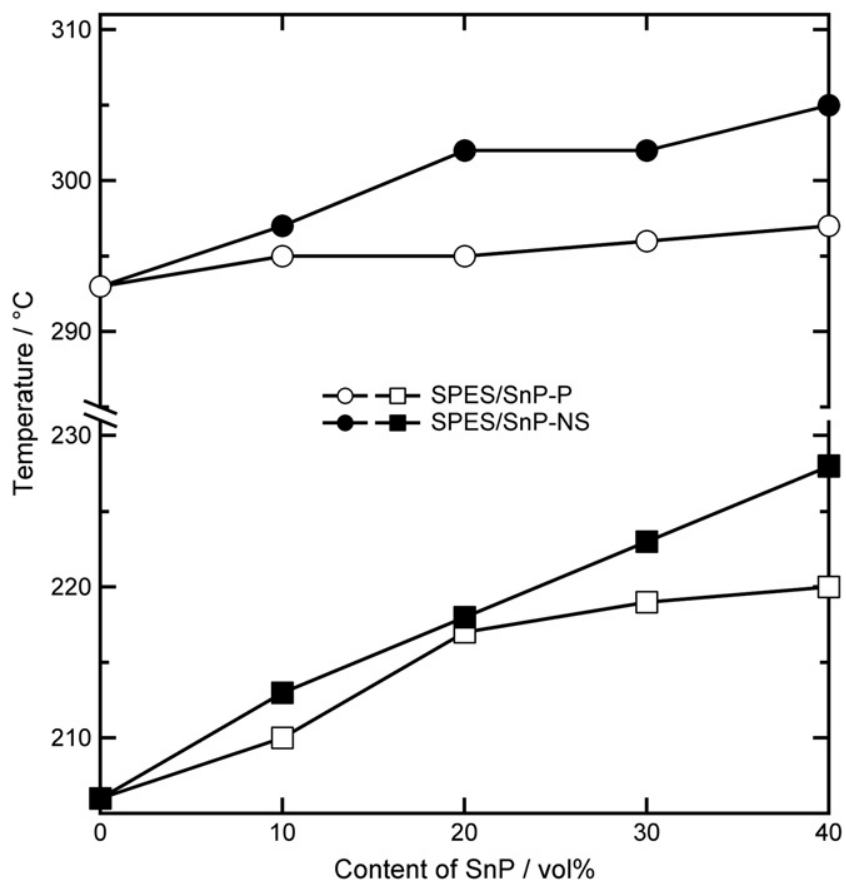

Fig. 7. Glass-transition temperatures and splitting-off temperatures of $\mathrm{SO}_{3} \mathrm{H}$ groups for SPES/SnP membranes. The square and circular symbols represent the glass-transition temperature and the splitting-off temperature, respectively.

conductivity, and the conductivity for SPES/SnP-NS decreased with increasing SnP content above 20 vol.\%. On the other hand, the conductivity for SPES/SnP-P membranes was almost independent of the SnP content, although SPES/SnP-P20vol.\% showed a slightly higher conductivity under $90 \%$ RH than did other SPES/SnP-P membranes.

It has been reported that the conductivity of SPEEK/ZrP-NS membranes can be improved by increasing the organic-inorganic interfacial area [29]. In the SPES/SnP membrane, we believe that water is strongly trapped in the SPES-SnP interfacial layer by

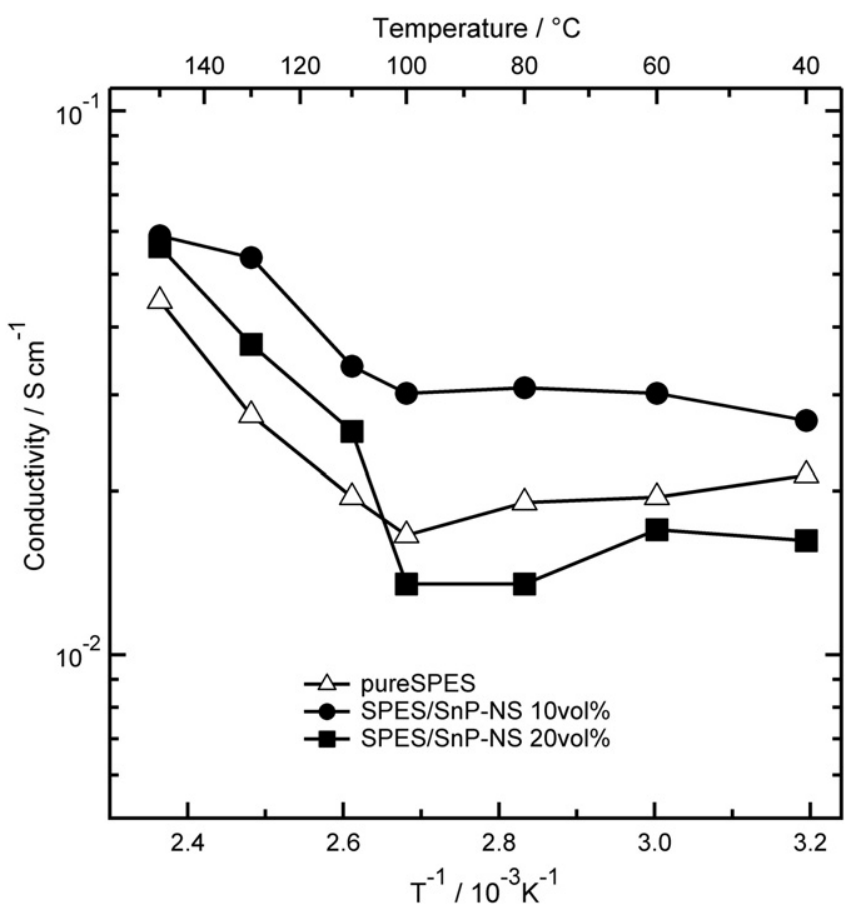

Fig. 8. Temperature dependence of the conductivity of SPES/SnP-NS with various SnP contents at saturated water vapor pressure. hydrogen bonds, and rapid proton transfer occurs through hopping in the hydrogen-bond network. Therefore, the increase in the SPESSnP interfacial area produced by using SnP-NS should lead to the formation of large numbers of connections between SPES-SnP interfacial layers and thereby to an increase in proton conductivity for SPES/ SnP-NS10vol.\% under 90\% RH. On the other hand, because of the small size of the SPES-SnP-P interfacial area, the hydrogen-bond network is probably intermittently connected in SPES/SnP-P10vol.\% and well connected in SPES/SnP-P20vol.\%.

At a lower $\mathrm{RH}$ of $30 \%$, the conductivity for SPES/SnP-NS decreased with increasing SnP content. As mentioned before, the water uptake decreased as a result of the stabilization of the membrane structure, and the effect of the stabilization was increased by using SnP-NS as the inorganic filler. We suggest that because of the low humidity, very little water is present in the SPES-SnP-NS interfacial layer, causing a decrease in conductivity even if the hydrogen-bond network is fully connected.

The changes in weight under various water vapor pressures at $130{ }^{\circ} \mathrm{C}$ are shown in Fig. 10. The samples were held at each pressure for $4 \mathrm{~h}$ until the weights became nearly constant. The weights of all the samples decreased with decreasing RH. The pure SPES membrane showed the greatest degree of dehydration, and the result was similar to that shown in Fig. 2. Fig. 11 shows the relationship between the conductivity and the amounts of water for pure SPES, SPES/ SnP-P10vol.\%, and SPES/SnP-NS10vol.\% membranes. The amounts of water were estimated from the weight decreases in Fig. 10, and the conductivities were measured for the three membranes at $130{ }^{\circ} \mathrm{C}$ under 20, 45, 70, and 95\% RH. At 70 and 95\% RH, the SPES/SnPNS10vol.\% showed the highest conductivity although it contained the least amount of water. However, at 20 and 45\% RH, SPES/SnP-NS10vol.\% showed the lowest conductivity and contained the least amount of water. It is likely that at high RH, the protons in the SPES-SnP interfacial layer can move faster through the hydrogen-bonded network as a proton-conducting pathway and that connections between hydrogenbond networks lead to an increase in conductivity. However, at low $\mathrm{RH}$, because of the very small amount of water in the SPES-SnP

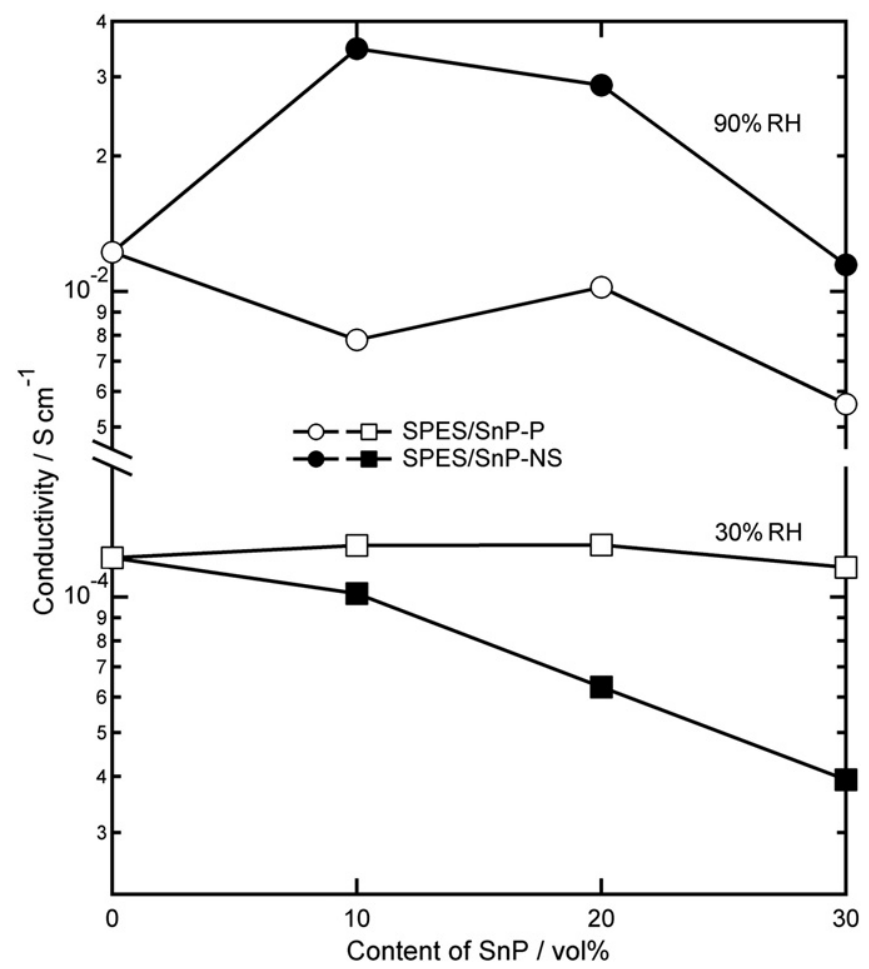

Fig. 9. Proton conductivities of SPES/SnP membranes with various SnP contents measured at $130{ }^{\circ} \mathrm{C}$ under $90 \%$ and $30 \%$ relative humidity. 


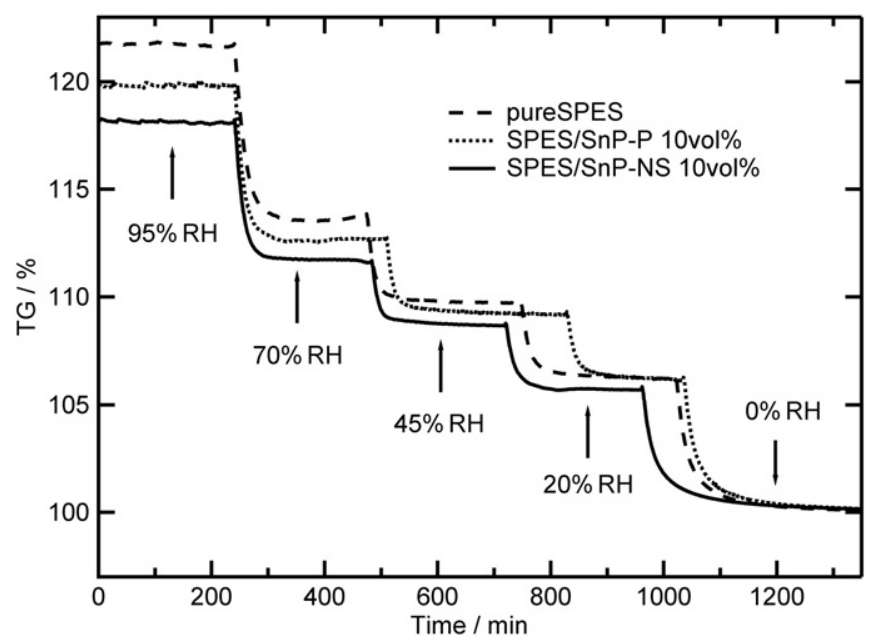

Fig. 10. Changes of weight with relative humidity as a function of holding time at $130{ }^{\circ} \mathrm{C}$ under $95 \%$ to $0 \%$ relative humidity.

interfacial layer, it is probable that the hydrogen-bonded network no longer functions effectively as a proton-conducting pathway. The conductivity of SPES/SnP-P10vol.\% was the lowest among the samples at 70 and 95\% RH, although the amount of water in SPES/SnP-P10vol.\% was larger than that in SPES/SnP-NS10vol.\%. In SPES/SnP-P10vol.\%, it is likely that the hydrogen-bonded network is intermittently connected, as described above (Fig. 9) and that protons can move rapidly through the SPES bulk region.

\section{Conclusions}

SPES/SnP membranes with various volume ratios of inorganic filler (0-40 vol.\%) were prepared and characterized. TGA/DTA studies and FT-IR spectroscopy showed that the chemical and thermal stabilities were improved by the addition of SnP to the SPES matrix. The effect of structural stabilization was more evident for SPES/SnP-NS membranes than for SPES/SnP-P membranes. We found that networks of hydrogen bonds are formed at the interface between SPES and SnP in the composite membranes, and that the larger interfacial area of SPES/SnP-NS

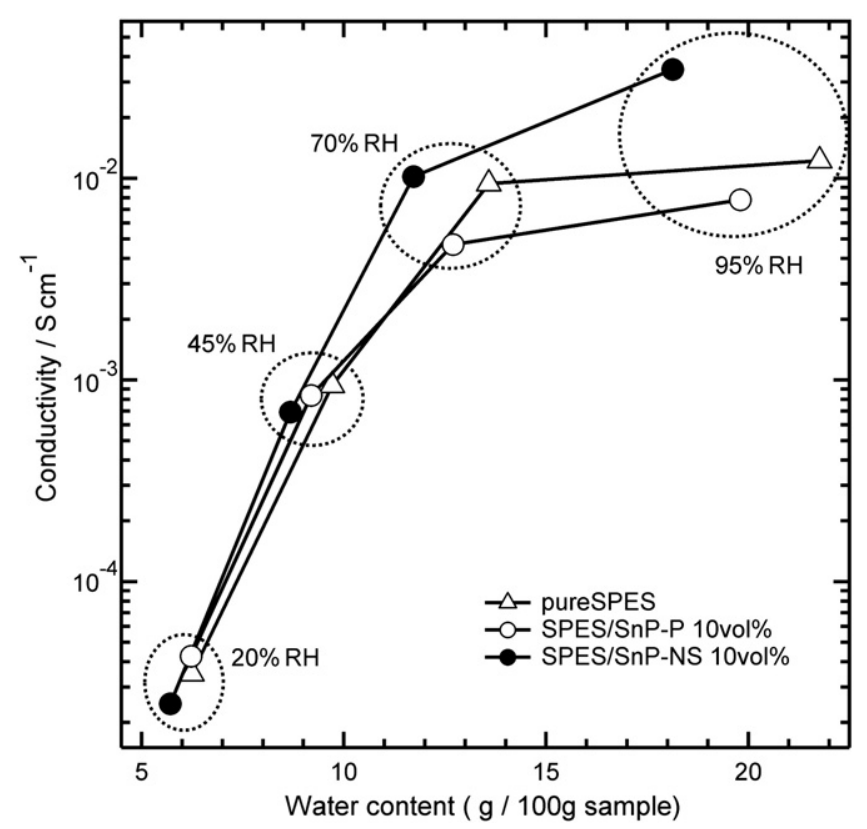

Fig. 11. Proton conductivities of membranes with various water contents estimated from the changes in weight shown in Fig. 10. membranes leads to increased chemical and structural stabilities compared with SPES/SnP-P membranes.

The proton-conducting properties of SPES/SnP-NS membranes were improved at high values of the RH. The degree of improvement in the conductivity of SPES/SnP-NS membranes was greater than that for SPES/SnP-P. At 95\% RH, the conductivity of SPES/SnP-NS10vol.\% was higher than those of pure SPES or SPES/SnP-P10vol.\%, although the amount of water present was smaller. We confirmed that the conductivity was improved by increasing the SPES-SnP interfacial area and the connectivity of the SPES-SnP interfacial layer, which operates effectively as a proton-conducting path.

At lower values of the $\mathrm{RH}$, however, the conductivity decreased with increasing SnP-NS content. We suggest that because of the very small amount of water in the SPES-SnP-NS interfacial layer, the hydrogen-bond network fails to operate effectively as a protonconducting pathway.

\section{Acknowledgments}

The authors are grateful to Toyota Motor Corporation for support of this research through the entrusted research project.

\section{References}

[1] Q. Li, R. He, J.O. Jensen, N.J. Bjerrum, Chem. Mater. 15 (2003) 4896-4915.

[2] G. Alberti, M. Casciola, L. Massinelli, B. Bauer, J. Membr. Sci. 185 (2001) 73-81.

[3] W.H.J. Hogarth, J.C. Diniz da Costa, G.Q. Lu, J. Power Sources 142 (2005) 223-237.

[4] K.D. Kreuer, J. Membr. Sci. 185 (2001) 29-39.

[5] Y. Yang, S. Holdcroft, Fuel Cells 5 (2005) 171-186.

[6] S.M.J. Zaidi, S.D. Mikhailenko, G.P. Robertson, M.D. Guiver, S. Kaliaguine, J. Membr. Sci. 173 (2000) 17-34

[7] F. Wang, M. Hickner, Q. Ji, W. Harrison, J. Mecham, T.A. Zawodzinski, J.E. McGrath, Macromol. Symp. 175 (2001) 387-396.

[8] F. Wang, M. Hickner, Y.S. Kim, T.A. Zawodzinski, J.E. McGrath, J. Membr. Sci. 197 (2002) 231-242.

[9] M.L. Di Vona, A. D'Epifanio, D. Marani, M. Trombetta, E. Traversa, S. Licoccia, J. Membr. Sci. 279 (2006) 186-191.

[10] M.L. Hill, Y.S. Kim, B.R. Einsla, J.E. McGrath, J. Membr. Sci. 283 (2006) 102-108.

[11] S.M.J. Zaidi, Electrochim. Acta 50 (2005) 4771-4777.

[12] B. Mecheri, A. D'Epifanio, M.L. Di Vona, E. Traversa, S. Licoccia, M. Miyayama, J. Electrochem. Soc. 153 (2006) A463-A467.

[13] S. Licoccia, E. Traversa, J. Power Sources 159 (2006) 12-20.

[14] J.-M. Thomassin, C. Pagnoulle, D. Bizzari, G. Caldarella, A. Germain, R. Jérôme, Solid State Ionics 177 (2006) 1137-1144.

[15] Y. Daiko, H. Sakamoto, K. Katagiri, H. Muto, M. Sakai, A. Matsuda, J. Electrochem. Soc. 155 (2008) B479-B482.

[16] M.M. Hasani-Sadrabadi, S.H. Emami, R. Ghaffarian, H. Moaddel, Energy Fuel 22 (2008) 2539-2542.

[17] Y.S. Kim, F. Wang, M. Hickner, T.A. Zawodzinski, J.E. McGrath, J. Membr. Sci. 212 (2003) 263-282.

[18] C.H. Lee, K.A. Min, H.B. Park, Y.T. Hong, B.O. Jung, Y.M. Lee, J. Membr. Sci. 303 (2007) 258-266.

[19] S. Wen, C. Gong, W.-C. Tsen, Y.-C. Shu, F.-C. Tsai, Int. J. Hydrogen Energy 34 (2009) 8982-8991.

[20] Y. Kawakami, M. Miyayama, Key Eng. Mater. 320 (2006) 267-270.

[21] G. Alberti, M. Casciola, U. Costantino, G. Levi, G. Ricciardi, J. Inorg. Nucl. Chem. 40 (1978) 533-537.

[22] D. Yoshimune, S. Sugata, S. Suzuki, M. Miyayama, J. Electrochem. Soc. 159 (2012) B91.

[23] Y. Kozawa, S. Suzuki, M. Miyayama, J. Electrochem. Soc. 156 (2009) B1401-B1405.

[24] Wang, Y. Omomo, N. Sakai, K. Fukuda, I. Nakai, Y. Ebina, K. Takada, M. Watanabe, T. Sasaki, Chem. Mater. 15 (2003) 2873-2878.

[25] T. Sasaki, M. Watanabe, J. Am. Chem. Soc. 120 (1998) 4682-4689.

[26] D.M. Kaschak, S.A. Johnson, D.E. Hooks, H.-N. Kim, M.D. Ward, T.E. Mallouk, J. Am. Chem. Soc. 120 (1998) 10887-10894.

[27] H.-C. Kuan, C.-S. Wu, C.-Y. Chen, Z.-Z. Yu, A. Dasari, Y.-W. Mai, Electrochem. Solid-State Lett. 9 (2006) A76-A79.

[28] D. Marani, A. D'Epifanio, E. Traversa, M. Miyayama, S. Licoccia, Chem. Mater. 22 (2009) 1126-1133.

[29] Y. Kozawa, S. Suzuki, M. Miyayama, T. Okumiya, E. Traversa, Solid State Ionics 181 (2010) 348-353.

[30] Y. Tanaka, S. Takano, M. Miyayama, J. Ceram. Soc. Jpn. 111 (2003) 323-326

[31] A.S. Arico, V. Baglio, A.D. Blasi, V. Antonucci, Electrochem. Commun. 5 (2003) 862-866.

[32] S.D. Mikhailenko, J. Zaidi, S. Kaliaguine, J. Chem. Soc. Faraday Trans. 94 (1998) 1613-1618.

[33] Y.S. Kim, F. Wang, M. Hickner, S. McCartney, Y.T. Hong, W. Harrison, T.A. Zawodzinski, J.E. McGrath, J. Polym. Sci. Part B: Polym. Phys. 41 (2003) 2816-2828.

[34] P. Krishnan, J.-S. Park, C.-S. Kim, J. Membr. Sci. 279 (2006) 220-229.

[35] M. Cappadonia, J.W. Erning, S.M. Saberi Niaki, U. Stimming, Solid State Ionics 77 (1995) 65-69. 